\title{
Klimawandel, Krankheiten und Konflikte
}

\author{
Lionel Jarvis ${ }^{a}$, \\ Hugh Montgomery ${ }^{b}$, \\ Neil Morisettic, Ian Gilmored \\ a Surgeon rear admiral, \\ UK Ministry of Defence, \\ London \\ b Director UCL Institute for \\ Public Health and \\ Performance, London \\ c Professor UK Ministry of \\ Defence, London \\ d Professor, Royal Liverpool \\ Hospital
}

Übersetzung: Ruth van der Zypen-Millard

Der englische Originaltext findet sich unter: www.bmj.com/content/342/ bmj.d1819.full

Korrespondenz:

Ruth van der Zypen-Millard Political and Public Affairs Officer

British Embassy

Thunstrasse 50

CH-3005 Bern

Tel. 0313597717
Sie stehen in einem gegenseitigen Verhältnis, und daher ist eine Kooperation medizinischer und militärischer Bereiche vonnöten. Krisen in der menschlichen Gesellschaft bedeuten Schaden an der menschlichen Gesundheit. Dieser ereignet sich auch ausserhalb von Kriegen. Ein kürzlich erschienener Bericht des International Institute for Strategic Studies (IISS) hat darauf hingewiesen, dass die Folgen des Klimawandels in der ersten Hälfte des 21. Jahrhunderts eine Bedrohung für die kollektive Sicherheit und der Weltordnung sein werden. Der begrenzte Zugang vieler Menschen zu Nahrung, sauberem Wasser, Energie, sanitären Einrichtungen und Gesundheitsversorgung wird zur Massenmigration und zum Wettbewerb um die verbleibenden Ressourcen führen. Die Häufigkeit von Unterernährung, Durchfall und ansteckenden Krankheiten wird zunehmen. Zudem werden die neonatale und auch die Erwachsenen-Mortalität steigen, als Folge von gewalttätigen Konflikten. So hatten z.B. 2004 sieben von zehn Ländern mit Konflikt- oder Post-Konfliktgesellschaften die höchste Mortalität von Kindern unter 5 Jahren.

Der IISS-Bericht bestätigt, dass der Klimawandel den zunehmenden $\mathrm{CO}_{2}$-Emissionen und anderen Treibhausgasen direkt zuzuschreiben ist. Er prognostiziert, dass extreme Wetterphänomene und Veränderungen der Wasser-Ressourcen die globale Nahrungsund Energieproduktion massiv beeinflussen werden. Der Direktor des US-amerikanischen National Intelligence, Dennis Blair, hat kürzlich gesagt, dass neuste Klimaforschung die rapide Zunahme von Folgen der Klimaveränderung aufzeigt. Auch der VierjahresBericht des US-Verteidigungsministeriums zuhanden des US-Parlaments bestätigt, dass die Klimaveränderung Armut, Verschlechterung von Umweltbedingungen und weitere Schwächung von fragilen Regierungen zur Folge haben wird. Weiter warnt der Bericht vor der Nahrungs- und Wasserknappheit, der Verbreitung von Seuchen und der daraus resultierenden Massenmigrationen, die ein Risiko für die nationale Sicherheit von Staaten werden könnten.

Auch ein Lancet-Bericht von 2009 hat betont, dass der Klimawandel die grösste globale gesundheitliche Bedrohung des 21. Jahrhunderts ist. Die gewichtigsten Bedrohungen kämen von den Folgen der Wasserund Nahrungsknappheit, des Mangels an Unterkunft und Siedlungen und der daraus folgenden Völkermigration. Dasselbe bestätigt auch der kürzlich erschienene IISS-Bericht; nach ihm besteht eine enge
Korrelation zwischen Konflikten und schlechter Gesundheit.

Derweil wächst die Bevölkerung der Erde weiterhin und wird gemäss Schätzungen bis im Jahr 2050 8,9 Milliarden erreichen. Der Anteil der städtischen Bevölkerung wird voraussichtlich bis zum Jahr 2030 von $13 \%$ (1990) auf 60\% ansteigen. Die zunehmend urbane Gesellschaft wird sich auf küstennahe Megacities konzentrieren (Städte mit über 10 Millionen Einwohnern) und wird sehr anfällig werden auf Ressourcenknappheit. Wenn nun extreme Wetterphänomene oder steigende Meeresspiegel in diesen fragilen Gesellschaften Schäden anrichten, könnte dies die öffentliche Gesundheit negativ beeinflussen. Aufgrund von Wasserknappheit, Mangel an sanitären Einrichtungen sowie an Heizung und Nahrung könnte es zur Verbreitung von Seuchen kommen.

Wie bei guter medizinischer Praxis üblich, ist die Prophylaxe von grösster Bedeutung. Der IISS-Bericht betont, dass es eine nachhaltige Investition in Infrastruktur und neue Technologien braucht und dass ein Wandel zur Nutzung von erneuerbaren Energien stattfinden muss.

Wir müssen unsere Städte und deren Infrastrukturen anpassen, um diesen Herausforderungen gerecht zu werden. Die Planung von Infrastruktur sollte einhergehen mit Einrichtungen zum Wohl der öffentlichen Gesundheit (z. B. Ausbau der Frischwasser- und Abwasseranlagen, Energiegewinnung aus Abfällen aller Art und den Bau von Strassen, um den Wasserabfluss zu gewährleisten). Wie bei anderen präventiven Massnahmen sollte auch hier ein von gesellschaftlicher und multinationaler Kooperation geprägter Ansatz zum Zug kommen.

Themen wie diese werden ausführlich diskutiert an der Konferenz «Climate change - how to secure our future wellbeing: a health and security perspective», die am 17. Oktober im British Medicine Association House in London durchgeführt wird. Ärzte werden oft als Autoritäten angesehen, und es wird ihnen Vertrauen entgegengebracht. Daher sollten sie sich Gehör verschaffen, damit man möglichst bald zur Tat schreitet.

Ausführliche Informationen über die Konferenz finden sich auf deren Webseite unter http://bit.ly/ climatechange2011. Weitere Informationen zum Thema unter http://resources.bmj.com/bmj/about$\mathrm{bmj} /$ media-partnerships/climate-change2014howto-secure-our-future-wellbeing-a-health-and-securityperspective 